sphere. Joss' model did not produce flashes if the temperature of the core was too high, although the core was still in thermal equilibrium with the time-averaged heat flow. X-rays are, of course, produced more efficiently by gravitational energy release when the matter is first accreted. The ratio of accretion luminosity to thermonuclear luminosity $L_{a} / L_{n}$ must be $\geqslant 30$, for conventional neutron star models, and $\sim 300$ for Joss' model. $L_{\mathrm{a}} / L_{\mathrm{u}}<25$ for MXB1759-29 and is $\leqslant 2$ for the rapid burster, if it is assumed that the accretion luminosity emerges as relatively continuous $\mathrm{X}$-rays over the same time interval as that on which bursts are observed. This tends to rule out thermonuclear flashes for the rapid bursts and may prove to be a problem for this model in general. Future work on X-ray bursts will one hopes improve our knowledge of neutron stars and thereby teach us some high energy physics.

\section{Gene conversion in mitochondria}

\section{from D. H. Williamson}

THE article on page 577 of this issue by Strausberg and his colleagues from Dallas will rekindle interest in a longstanding problem in the mitochondrial field, namely the mechanism of recombination between mitochondrial DNA molecules.

Mitochondrial genetics is dominated by work on bakers' yeast. and has been ever since the discovery in this organism, some 15 years ago, of the first mitochondrial point mutations conferring resistance to either chloramphenicol or erythromycin. These drugs block protein synthesis on mitochondrial ribosomes, and it happened that these first resistant mutants all mapped close together in the region we now know specifies the $21 \mathrm{~S}$ mitoribosomal RNA. These mutants were the first tools with which mitochondrial recombination in crosses could be studied, and quite early on, a puzzling phenomenon emerged; in certain crosses, a marked polarity was observed amongst the recombinants, alleles carried by one parent being greatly favoured at the expense of those represented by the other. This polarity was not observed in all crosses, and it was soon realised that any strain could formally be categorised as carrying a hypothetical determinant called omega $(\omega)$ in one or other of two configura-

D. H. Williamson is at the National Institute for Medical Research, Mill Hill. tions, $\omega^{+}$and $\omega^{-}$. Polarity of recombination was observed only in $\omega^{+} \times \omega^{-}$ crosses, and not in either of the other two pairwise combinations. The $\omega$ determinant was independent of the mating type of the cell, but an early idea was that its role was a sexual one analogous to $\mathrm{Hfr}$ in bacteria and, for a while, the notion of mitochondrial sex was in vogue. However, when mutants in other unlinked genes were discovered, making four-point crosses feasible, it became clear that the analogy with bacterial sex was invalid. and in 1974 the Gif group (Dujon, Slonimski \& Weill Genetics, 78, 415; 1974) proposed a comprehensive model to account for all the facts then known. Esentially their model supposes that the mitochondrial DNA molecules in pairs of mating cells undergo repeated rounds of recombination. At the level of the individual molecules, recombination events are considered always to be non-reciprocal. However at most loci, gene conversion can go randomly in either direction. thus generating equal numbers of reciprocal recombinant types in the population as a whole, and obscuring the nonreciprocal nature of the events at the molecular level. At the $\omega$ locus, on the other hand, the conversion is seen as always occurring in one direction, effectively favouring alleles of the $\omega^{+}$ parent, and at the same time unmasking the asymmetric nature of the molecular interactions.

We have since learnt that as far as the events at the $\omega$ region are concerned, this model is broadly correct. It has recently been shown that the 21S RNA gene of $\omega^{+}$strains carries an insertion of about 1,000 base pairs which is absent from $\omega^{-}$strains. It is this insertion that seems to be responsible for 'directing' the conversion events in its immediate vicinity. Until now, however, no comparable phenomenon had been discovered in other regions of the genome, and the events at $\omega$ were coming to be regarded as something of an odd-ball.

In the light of today's article from the Dallas group, this view becomes a shade less tenable. This group reports on a rather similar state of affairs affecting a recently discovered mitochondrial gene called var 1 . This gene owes its name to the fact that its polypeptide product varies in size in different strains, the extent of this variation amounting to some 4,000 daltons. or around $10 \%$ of the polypeptides' molecular weight. The work on varl is of unusual technical interest, since its function is completely unknown, and all the genetic analyses have revolved round the ingenious application of gel electrophoresis to bulk mating populations. With the aid of a variety of genetic and physical mapping pro- cedures, the Dallas group have already precisely located the varl gene on the map, and have shown that the altered sizes of the variant forms of the polypeptide directly reflect changes in the size of the structural gene itself.

The current paper reports on the behaviour of different varl alleles in crosses. It shows, rather clearly, that alterations in the size of the gene (and hence of its polypeptide product) arise as a result of asymmetric gene conversion events involving two small DNA segments (about 36 and 57 base pairs long) which can be independently inserted. by recombination, into alleles which lack them. The very demonstration of these events in a system in which phenotypic expression is restricted to the rate of migration of protein molecules in a gel, is something of a tour de force, and fully deserves our admiration. However, the observations have a wider significance in that the phenomena look rather similar to those seen at the $\omega$ region. In both cases the gene conversion events occur at high frequencies, and both regions possess insertions presumably responsible for 'directing' the nonreciprocal recombination events at the locus, thereby making them detectable in the overall molecular population.

The main conclusion is clear. Gene conversion is not restricted to the $\omega$ locus, and its occurrence at other regions of the genome may have been missed before now simply because of the lack of strategically placed pairs of markers to make it visible. Although no other protein products have been observed to behave like varl, there is no lack of evidence for other insertions in the genome (see the Strausberg article for references). Nor is there any lack of models embodying gene conversion to explain some unresolved problems in the transmission genetics of this system. Evidence such as that presented on page 577 begins to invest such models with an air of respectability.

\section{The first division}

\section{from Clive Lloyd}

Protopiasts prepared from tobacco leaves now form the staple experimental dict of investigators studying viral infection. somatic hybridisation. wall synthesis, properties of the plasma membrane-the list goes on-but the intriguing fact goes almost unnoticed that in the process of removing the cell wall, non-dividing leaf cells are stimulated to divide. For instance. remove palisade cells from tobacco 